\title{
Nanovoid Relaxation in a Series of Copolyester Glasses Under Cyclic Loading Using Synchronous PALS
}

\author{
NANJIAN SUN, ${ }^{1}$ JIANWEI LIU, ${ }^{1}$ TERRY DULL, ${ }^{2}$ ALBERT F. YEE ${ }^{1,3}$ \\ ${ }^{1}$ Macromolecular Science and Engineering Center, University of Michigan, Ann Arbor, Michigan 48109 \\ ${ }^{2}$ Department of Physics, University of Michigan, Ann Arbor, Michigan 48109 \\ ${ }^{3}$ Department of Chemical Engineering and Materials Science, University of California, Irvine, California 92697
}

Received 6 December 2005; revised 19 March 2006; accepted 22 March 2006

DOI: 10.1002 / polb.21168

Published online in Wiley InterScience (www.interscience.wiley.com).

\begin{abstract}
This study examines if correlation between a specific molecular motion and the growth and relaxation of nanovoids, which are precursors to crazes, can be established. A novel technique, positronium annihilation lifetime spectroscopy (PALS) synchronized with cyclic stress, is reported. In this technique the positronium annihilation signal was accumulated in sixteen channels corresponding to sixteen phases of the sinusoidal load, which allowed statistically significant data for nanovoid generation and relaxation due to the fluctuating stress to be accumulated. This technique was applied to a series of copolymers of poly(ethylene terephthalate) (PET) and poly(1,4-cyclohexylenedimethylene terephthalate) (PCT). Previous studies have shown that the cyclohexylene rings in the main chain of PCT actively undergo chair-boat-chair conformational transitions in the glassy PCT at around room temperature. The PET-co-PCT series was also chosen for this study because the crazing stress increases systematically with the cyclohexylene content. The synchronized PALS data are consistent with the hypothesis that transient nanovoids generated by the cyclic stress in polymers containing more cyclohexylene rings relax more readily than those with fewer rings. The results also correlate well with the crazing stress in this series of copolymers. @ 2007 Wiley Periodicals, Inc. J Polym Sci Part B: Polym Phys 45: 1410-1417, 2007
\end{abstract}

Keywords: crazing; polyesters; relaxation; toughness

\section{INTRODUCTION}

The correlation between secondary mechanical relaxations and mechanical properties of polymers has been a subject of intense interest since the $1960 \mathrm{~s} .{ }^{1-4}$ These studies involve hypotheses on the role of molecular motions on the yield behavior of polymers. Interestingly, no hypothesis has been advanced on the possible role of molecular motions in crazing until the series of publications $s^{5-7}$ on the

Correspondence to: A. F. Yee (E-mail: afyee@uci.edu) Journal of Polymer Science: Part B: Polymer Physics, Vol. 45, 1410-1417 (2007) ○2007 Wiley Periodicals, Inc.

1410 copolymer series based on poly(ethylene terephthalate) (PET) and poly(1,4-cyclohexylenedimethylene terephthalate) (PCT) appeared. The purpose of this article is to see if a firm correlation between a specific molecular motion and the growth and relaxation of nanovoids, which are precursors to crazes, can be established. A novel technique for observing the dynamic growth and relaxation of these nanovoids under cyclic loading conditions is also reported.

Chen et al. ${ }^{5,6}$ studied the deformation behavior of PET-co-PCT and found that yield stress decreased, whereas craze stress increased with cyclohexylene (C-ring) content in the main chain of 
the copolymers, and that the activation volume in yielding based on Eyring's model increased with C-ring content, while the activation energy did not exhibit any significant change. They proposed that when C-rings are inserted into a copolymer's main chain, these rings can undergo chair-boat-chair conformational transitions which are observable as secondary relaxations. They suggested that such conformational transitions could induce translational motions of the attached terephthalate groups, thus giving rise to longer range molecular motions and further facilitating macroscopic shear yielding. They further proposed, for the first time, that the chain motions also suppress craze nucleation. Exactly how the suppression mechanism might work was not elaborated on. Liu and Yee, ${ }^{8}$ working with a series of polyestercarbonates that also contain the aforementioned C-rings, hypothesized that high chain mobility due to conformational transitions help to collapse nanovoids that are precursors to stable craze nuclei and increase the stress necessary for the formation of stable nuclei in craze initiation. More recently this hypothesis was examined by $\mathrm{Li}$ and $\mathrm{Yee}^{7}$ who investigated the growth of nanovoids in the same series of PET-co-PCT copolymers as studied by Chen et al. under constant tensile strain energy conditions. They showed that the scale of local segmental motion did affect nanovoid growth, which should be the precursory step to craze formation. They used coupled stress-PALS tests to show that nanovoids in copolymers containing higher fractions of C-rings exhibited a lower tendency to grow than those with lower fractions. To explain these observations, they suggested, among other things, that larger scale local segmental motion results in slower kinetics in nanovoid growth, which might retard craze nucleation and increase craze stress. Their suggestion is consistent with the hypothesis of Liu and Yee, but do not prove it.

To more directly support the Liu-Yee hypothesis, it is necessary to show that the more chain mobility there is, the easier it is for nanovoids created by the application of a tensile stress, that is, a stress state where the hydrostatic component is dilatative, to relax or collapse. If this is indeed the case, then nanovoid growth and collapse will closely track the application of stress; if not, then they will lag behind the stress. One experiment to test this hypothesis, akin to a dynamic mechanical test, is to apply a cyclic stress, while monitoring the growth and collapse of nanovoids, using PALS; furthermore, the experiment must be conducted in conditions wherein the segmental motion is active.

\section{Experimental Approach}

PALS, which stands for Positronium Annihilation Lifetime Spectroscopy, is an ideal technique for probing nanovoid growth and collapse because motions by molecular segments are expected to generate voids in the PALS sensitivity range, of 2$20 \AA$ inside nonconducting materials. ${ }^{9-11}$ When positrons are injected into a material, positronium atoms can be formed. Two states, ortho-positronium (o-Ps) and para-positronium ( $p$-Ps), depending on mutual spin orientation of the electron and the positron, are possible. For our purposes, the more important state is $o$-Ps. The $o-\mathrm{Ps}$ is concentrated in "holes" within the material under study, the lifetime of which $\left(\tau_{3}\right)$ is a function of the hole size and the intensity of which $\left(I_{3}\right)$ a function of the number of holes. A detailed analysis of $\tau_{3}$ and $I_{3}$ can give an estimate of hole size and total hole volume.

Because the hole volume change could be directly related to the microscopic molecular motion, a number of investigations of mechanical properties of glassy polymers have been carried out using PALS. ${ }^{8,12-16}$ In most of these investigations the specimens were held near room temperature at fixed levels of strain, while PALS data were collected ${ }^{8,15-17}$ and are properly described as static investigations. It takes at least $30 \mathrm{~min}$, and often more, to collect each data point during a static PALS experiment. The dynamics of hole size and population changes with relaxation times short when compared with a PALS data collection interval are thus unobservable in static investigations. While the asymptotic or static characteristics of stress-induced hole size and population changes provide valuable information, the dynamic characteristics are expected to be the most revealing in understanding the role local segmental motions play in hole evolution. If dynamic information were available it would presumably be possible to determine if molecular motion facilitates the generation and collapse of transient volume on the microscopic scale during applied dilatative stress. To monitor the dynamic or transient behavior of holes under imposed stress, it is necessary to employ a dynamic mechanical test coupled with a dynamic PALS measurement. The idea is to study the evolution of the hole size and population with PALS as the material is cyclically loaded. In such a study the time period of the load oscillation must be small when compared with the characteristic time of the hole relaxation so that the dynamic part of hole growth and relaxation can be observed. But such a coupled 


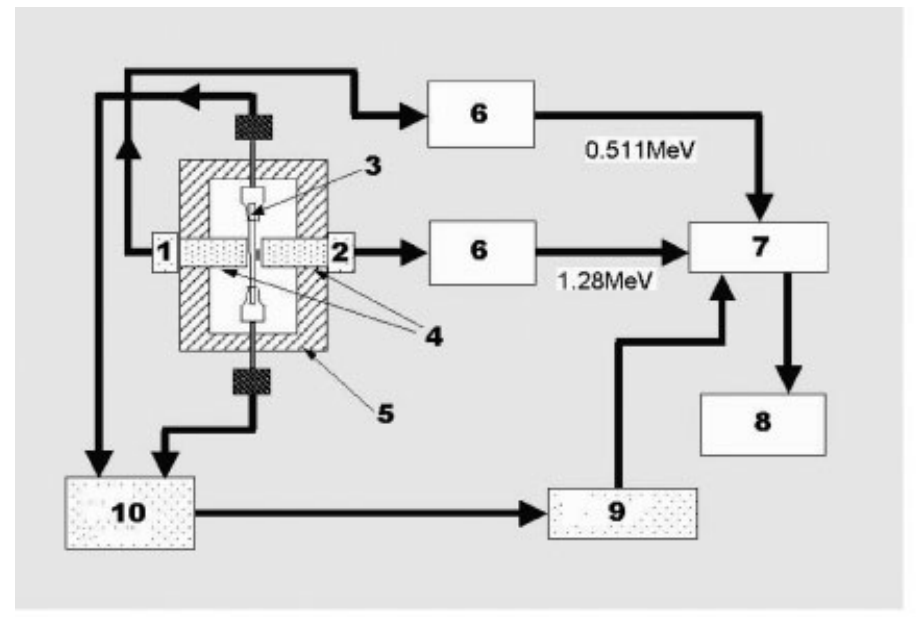

1. Start, 2. Stop, 3. Specimen, 4. Scintillator and photomultiplier tube, 5.Instron test chamber, 6. Constant fraction differential discriminator, 7. Multichannel analyzer, 8. Computer workstation, 9. Synchronization device, 10. Instron controlling computer

(a)

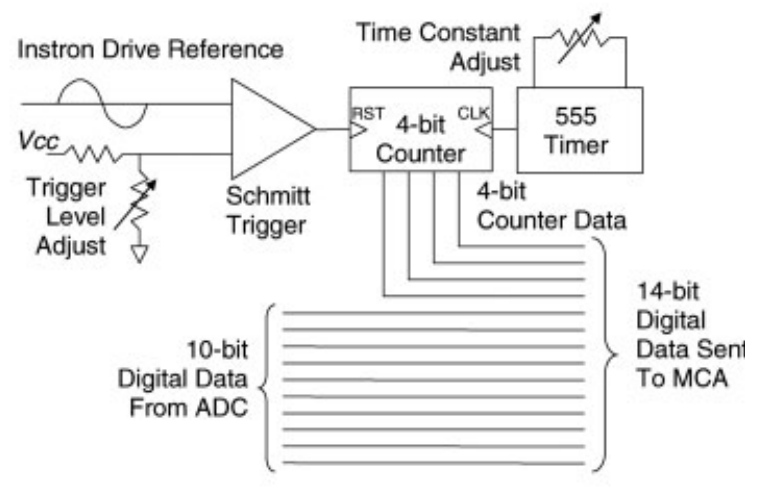

(b)

Figure 1. (a) Schematic plot of synchronous PALS coupled cyclic stress test system; (b) PALS synchronization circuit.

test is very difficult to perform because the PALS data accumulation time using a typical radiation source of micro-Curie strength is orders of magnitude larger than characteristic relaxation times. In this article we describe a technique that circumvents this difficulty and present some preliminary results.

To monitor the transient behavior of holes under cyclic stress, a dynamic PALS technique, viz., PALS synchronized with cyclic loading, has been developed and is used in the present research.

In this technique the signal used to drive the servohydraulic mechanical test machine is also used to "code" the PALS data such that sixteen PALS spectra are simultaneously acquired, each associated with a different portion of the stress waveform. By applying cyclic loading over many cycles, statistically valid data can be obtained.

\section{EXPERIMENTAL}

\section{Specimens}

The specimens were supplied by Eastman Chemical in the form of injection molded tensile bars. The dimension of the dogbone-shaped sample bars was $65 \times 12.7 \times 3.2 \mathrm{~mm}^{3}$ (length $\times$ width $\times$ thickness). The molecular weights and molding temperatures of these copolymers are listed elsewhere. ${ }^{6}$ All the specimens were transparent and tested amorphous by X-ray. A 12.7-mm gauge length extensometer was clipped to the gauge section of each specimen to provide the strain signal for feedback control needed by the servohydraulic Instron.

\section{Sinusoidal Cyclic Loading Combined Synchronous PALS System}

An Instron 1332 servohydraulic mechanical test machine operated under the strain control mode was used to produce sinusoidal cyclic strain. The sample, testing machine and PALS system are schematically shown in Figure 1(a). The sinusoidal signal from the testing machine used to control the cyclic strain was also used as a time reference for the PALS synchronization circuit shown in Figure 1(b).

The peak-to-peak strain in sinusoidal cyclic loading in the present experiments ranged from 0 to $1 \%$, which meant that the specimen was always under tension. Keeping the strain to a maximum of $1 \%$ assured a sufficiently long fatigue lifetime (which is referred to herein as the endurance lifetime to distinguish it from the positronium lifetime) under $1-10 \mathrm{~Hz}$ cyclic loading, while at the same time produced a relatively high hole volume change, resulting in a high signal to noise ratio for the PALS data.

The temperature control equipment is shown in Figure 2(a,b). A specimen passing through an aluminum chamber was clamped in the testing machine. On the specimen a $25 \mu \mathrm{Ci}{ }^{22} \mathrm{NaCl}$ positron source, which generated 2300 counts per sec-

Journal of Polymer Science: Part B: Polymer Physics DOI 10.1002/polb 


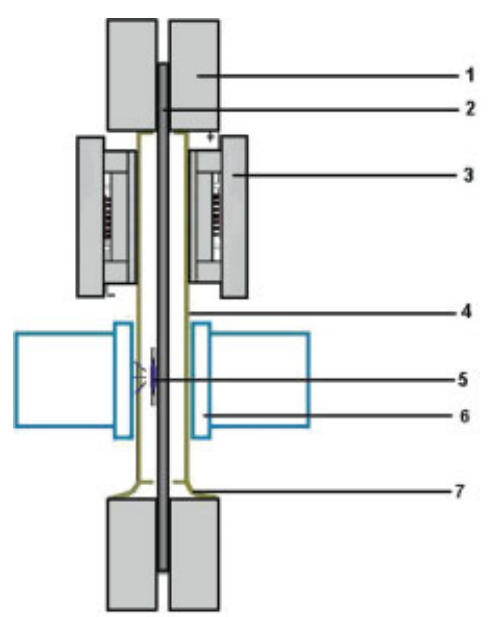

(a)

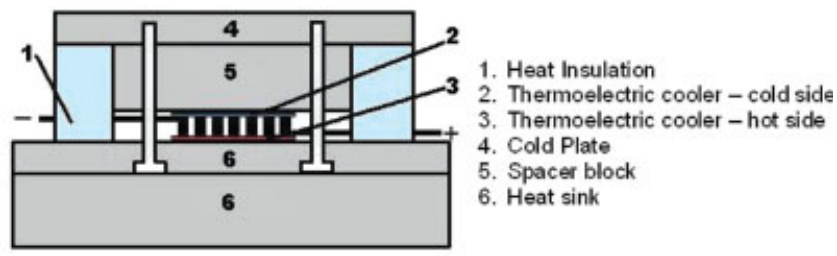

(b)

Figure 2. (a) Assembly of sample cooling, mechanical testing, and PALS data detection system; (b) Assembly of thermoelectric cooler. [Color figure can be viewed in the online issue, which is available at www.interscience.wiley.com]

ond was attached. Two thermoelectric coolers (TEC), manufactured by Melcor, were positioned near the chamber's upper position to cool the sample chamber. Passing DC current through the TEC generates a temperature differential across the device of at least $70{ }^{\circ} \mathrm{C}$. In the present experiments, the cool side of TEC was attached to the sample chamber; the hot sides were attached to a heat sink and fan to remove the excess heat while cooling the chamber. The chamber was then coated with polyurethane foam to provide thermal insulation. Since the TEC was very thin, $\sim 3 \mathrm{~mm}$, the whole cooling system was highly compact, and the distance between the scintillator and the positron source could be kept very small, less than $20 \mathrm{~mm}$, which allowed a high coincidence rate in PALS data acquisition. The upper end of the chamber was tightly attached to the upper static clamp, while a $\sim 5$-mm gap between the lower end of the chamber and lower clamp was maintained to allow movement of the lower clamp during cyclic loading. With this arrangement the sample could be cooled to $0.0 \pm 1.5^{\circ} \mathrm{C}$ or lower. It was also capable of keeping the scintillators at room temperature, which is necessary for proper performance.

The positron lifetime spectra were obtained by a conventional fast-timing coincidence method. Signals from the start and stop detectors, consisting of scintillators coupled to photomultipliers (Amperex XP2020 with S563 bases), were processed by two constant fraction differential discriminators (CFDD, Ortec583) with the start energy window normally set for the ${ }^{22} \mathrm{Na} 1.28 \mathrm{MeV} \gamma$-ray (indi- cating the birth of a positron) and the stop set for the $0.511 \mathrm{MeV}$ annihilation $\gamma$-rays. The time interval between the start and stop signals from the CFDDs was converted to an output pulse whose height is proportional to the time interval by a Time-to-Amplitude Converter (TAC, Ortec567). The TAC output pulses were then digitized by an analog-to-digital converter (ADC, ND581), combined with digital data from the PALS synchronization circuit, and recorded by an integrated multichannel analyzer (MCA, ND9900) through an ethernet compatible module (AIM, ND556) into a micro VAX 3100 workstation that performed both data acquisition and analysis.

The PALS synchronization circuit used a Schmitt trigger to detect the rising edge of the sinusoidal Instron signal. A 4-bit counter, driven by a 555 timing chip and reset by the rising edge of the Schmitt trigger output was used to count to sixteen. The time constant of the 555 was adjusted for each experiment so that sixteen pulses just covered one sinusoidal period. The 4-bit digital output of the counter was combined with the 10-bit digital output of the ADC to create 14-bit words recorded by the MCA. Using this technique sixteen PALS spectra could be simultaneously recorded with data in each spectra being accumulated in phase with a different portion of the cyclic strain waveform. The information derived from PALS could therefore be broken up into sixteen time channels encompassing the stress sinusoid and transient characteristics of hole-size under cyclic loading could be studied. 
Table 1. Endurance Time of Copolyester Samples with Different C-Ring Contents Under 10 Hz Cyclic Loading Strain of $1 \%$

\begin{tabular}{lccccc}
\hline \multicolumn{1}{c}{ Sample } & PET & $\begin{array}{c}\text { EC3 } \\
(31 \% \text { C-Ring })\end{array}$ & $\begin{array}{c}\text { EC4 } \\
(62 \% \text { C-Ring })\end{array}$ & $\begin{array}{c}\text { EC5 } \\
(81 \% \text { C-Ring })\end{array}$ & PCT \\
\hline Endurance time $\left(25^{\circ} \mathrm{C}\right)$ & $>6$ days & $\sim 15 \mathrm{~h}$ & $\sim 30 \mathrm{~h}$ & $\sim 6$ days & $>6$ days \\
Endurance time $\left(0^{\circ} \mathrm{C}\right)$ & $5 \sim 6$ days & $<10 \mathrm{~h}$ & $\sim 15 \mathrm{~h}$ & $\sim 2$ days & 5 days \\
\hline
\end{tabular}

Temperature $=25 \pm 1.5^{\circ} \mathrm{C}$.

For the purpose of this synchronous PALS experiment, the most important results are $\tau_{3}$ and $I_{3}$. Through the following equation, $R$ (diameter of void) can be deduced: ${ }^{16}$

$$
\begin{gathered}
\tau_{3}^{-1}=\bar{\lambda}_{\text {spin }} G+\lambda_{o-\mathrm{Ps}}(1-G) \\
G=1-\frac{R}{R+\Delta R}+\frac{1}{2 \pi} \sin \frac{2 \pi R}{R+\Delta R}
\end{gathered}
$$

where $\lambda_{\text {spin }}=[2+0.75(1 / 142)] \mathrm{ns}^{-1}$ is the spinaveraged annihilation rate, $\lambda_{o-\mathrm{Ps}}=1 / 142 \mathrm{~ns}^{-1}$ is the vacuum decay rate of $o-\mathrm{Ps}, \mathrm{G}$ is the quantum mechanical probability that $o$-Ps is in the electron cloud, and $\Delta R=1.612 \AA^{18-20}$ is the $o$-Ps penetration depth into the wall of the hole wherein the $o$ Ps annihilates at the spin-averaged rate.

The relative intensity $I_{3}$ is commonly assumed to be proportional to the number density of the holes. The total hole volume is then proportional to the product of the average volume of a single hole volume $\frac{4 \pi}{3} R^{3}$ and $I_{3}$.

\section{RESULTS AND DISCUSSION}

Since the maximum strain used in the cyclic loading was relatively small $(<1 \%)$, the hole volume change was correspondingly small. In addition, PALS signals were distributed into 16-data channels, which further reduced the signal rate in each of these channels. These two factors resulted in a low signal-to-noise ratio that had to be compensated for, which was accomplished by extending the static PALS data acquisition time from several hours to about 6 days.

Not all the PET-PCT copolymer samples have fatigue endurance times longer than 6 days under cyclic loading, thus it was impossible to obtain complete data sets for all the materials. And these endurance times varied significantly with different C-ring contents (see Table 1). The reason is complicated. Endurance time is generally determined by the progressive growth of cracks as well as mechanical damping effects. ${ }^{21-24}$ Since endurance time decreases with increasing stress, factors that increase the strength of polymers also tend to increase endurance time. Endurance time is also enhanced by factors which suppress craze formation. Previous results ${ }^{6}$ showed that increasing Cring content in PET's main chain decreased the yield strength slightly and suppressed craze formation. Since these two results have opposite effects on endurance time, it is not surprising that no simple correlation between C-ring content and the endurance times of the materials studied can be found in Table 1. Another important factor is of course the fact that crazes tend to initiate on surface imperfections, and each of the specimens naturally contained a population of such surface flaws, some of which eventually nucleated crazes that grew to become fatal cracks.

As endurance time decreases with decreasing experimental temperature, synchronous PALS experiments were possible only on PET, EC5 and $\mathrm{PCT}$ at both 0 and $25^{\circ} \mathrm{C}$. Higher temperature runs were not performed because the relaxations are expected to be faster, making it difficult to observe any of the desired effects.

A typical synchronous PALS result under $10 \mathrm{~Hz}$ cyclic loading at $0{ }^{\circ} \mathrm{C}$ is shown in Figure 3 . The hole volume change of PET is fit to a sinusoidal function, which reveals a phase lag relative to the macroscopic strain of $23.5^{\circ}$ (with $\pm 4^{\circ}$ uncertainty). Phase lag of such a large magnitude is somewhat surprising since at $0{ }^{\circ} \mathrm{C}$ the $\gamma$-relaxational motion of PET is activated, with characteristic times much shorter than $0.1 \mathrm{~s}$. And furthermore the macroscopic command strain and response stress have no significant phase lag. Another interesting observation is that the maximum hole volume change is about $5 \%$, much larger than the maximum tensile strain of $1 \%$ which would result in a homogeneous dilatative strain of about $0.0 .2 \%$ (assuming Poisson's ratio $\sim 0.4$ ), which suggests that the maximum volume dilatation is very localized. This hole 


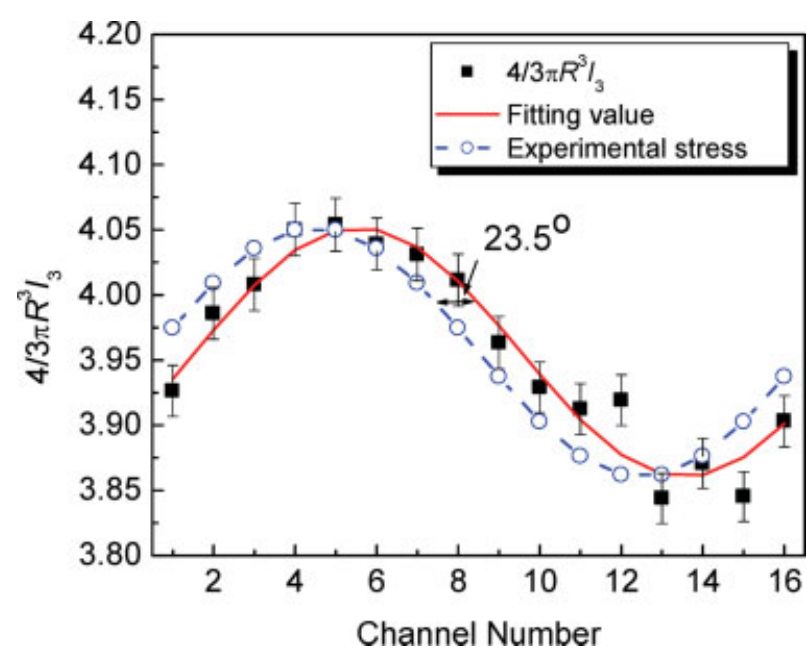

Figure 3. Plot of ${ }^{4} / 3 \pi R^{3} I_{3}$ versus Channel number for pure PET under $10 \mathrm{~Hz}$ cyclic loading at $0{ }^{\circ} \mathrm{C}$, amplitude of strain $=1 \%$. [Color figure can be viewed in the online issue, which is available at www.interscience.wiley.com]

volume change is transient in nature because it disappears when the strain is removed. In comparison, Ruan et al. found ${ }^{17}$ that the application of tensile strain up to $\sim 4 \%$ produced an increase in $\tau_{3}$ and total hole volume, but did little to change $I_{3}$. Hasan et al. ${ }^{15}$ found that before and after uniaxial compression, $I_{3}$ was unchanged by the applied strain, while $\tau_{3}$, and hence total hole volume, increased. More recently, Xie et al. ${ }^{16}$ conducted a stress-coupled PALS test on BPA-PC. They also found that mechanical stress affected mainly the size of existing holes, but did not generate or eliminate holes in the quasi-elastic deformation region. It should be pointed out that the results cited earlier were obtained in quasi-static step loading, not cyclic loading.

$I_{3}$ and $\tau_{3}$ results of PET under $10 \mathrm{~Hz}$ cyclic loading at $0{ }^{\circ} \mathrm{C}$ are shown in Figure 4, from which no apparent trend can be discerned. This is mainly due to the additional error introduced by the $I_{3}$ and $\tau_{3}$ curve fitting process. Specifically, during curving fitting, $I_{3}$ and $\tau_{3}$ are inversely correlated to each other, so that if the fitting process introduces a positive deviation for $I_{3}$, it will at the same time introduce negative deviations for $\tau_{3}$. As a result, errors for separate $I_{3}$ and $\tau_{3}$ are larger than the product [3/4] $\pi R^{3} I_{3}$. Consequently $I_{3}$ and $\tau_{3}$ results are not discussed separately in the following.

Figures 5 and 6 show that when the frequency of cyclic loading decreases to $3 \mathrm{~Hz}$ and further to 1 $\mathrm{Hz}$, the phase lag for PET decreases to $12.0^{\circ} \pm 4.9^{\circ}$ and $4.1^{\circ} \pm 5.7^{\circ}$, respectively. At the lowest frequency the uncertainty is comparable to the meas-

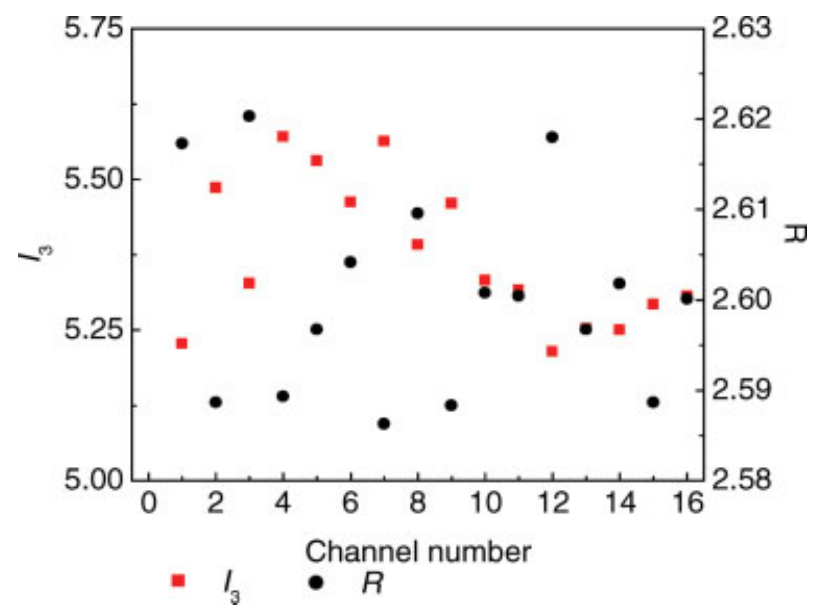

Figure 4. Plot of $I_{3}$ and $R$ versus Channel number for pure PET under $10 \mathrm{~Hz}$ cyclic loading at $0{ }^{\circ} \mathrm{C}$, amplitude of strain $=1 \%$. [Color figure can be viewed in the online issue, which is available at www.interscience.wiley.com]

ured phase lag so that the latter should be considered to be inconsequential. Meanwhile, the maximum hole amplitude also decreases from 0.082 \pm 0.007 to $0.04 \pm 0.004$. These observations are discussed along with the data in Tables 2 and 3.

According to the principle of time-temperature equivalence, increasing temperature has an effect akin to lowering the frequency because molecular relaxation is faster at higher temperatures. Consequently, both phase angle and amplitude are expected to decrease at higher temperatures and lower frequencies. It can be seen in Tables 2 and 3 that for the same sample, changes in both phase lag and amplitude of hole volume are consistent with this expectation. This was found to be true for

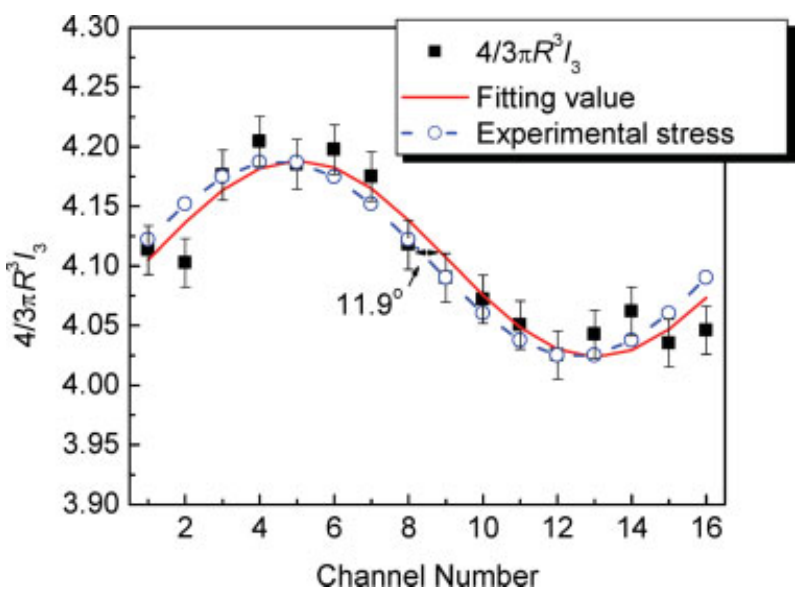

Figure 5. Plot of $4 /{ }_{3} \pi R^{3} I_{3}$ versus Channel number for pure PET under $3 \mathrm{~Hz}$ cyclic loading at $0{ }^{\circ} \mathrm{C}$, amplitude of strain $=1 \%$. [Color figure can be viewed in the online issue, which is available at www.interscience.wiley.com] 


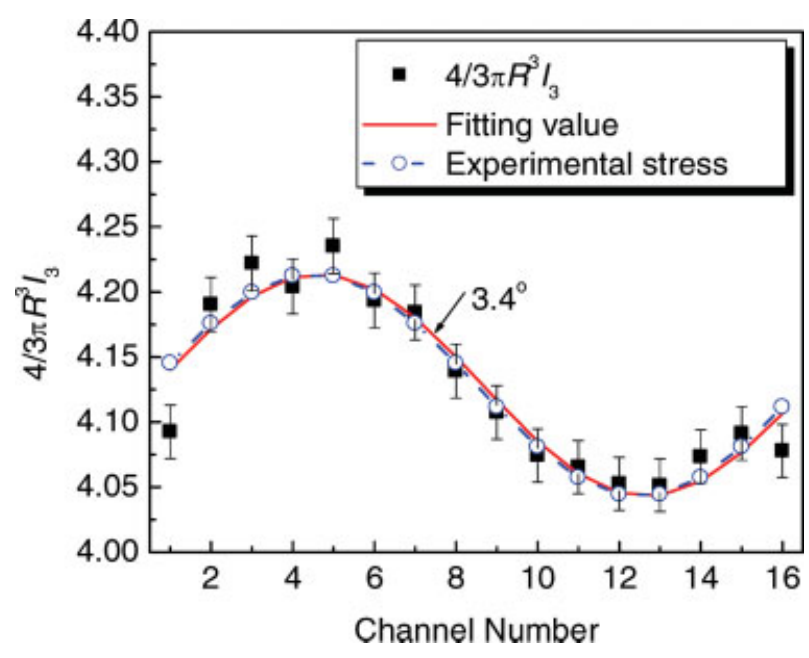

Figure 6. Plot of $4 /{ }_{3} \pi R^{3} I_{3}$ versus Channel number for pure PET under $1 \mathrm{~Hz}$ cyclic loading at $0{ }^{\circ} \mathrm{C}$, amplitude of strain $=1 \%$. [Color figure can be viewed in the online issue, which is available at www.interscience.wiley.com]

PET, EC5, and PCT. Note that in Table 3 (see values under phase lag in degrees), the phase lags of $\mathrm{PCT}$ at both frequencies are small negative values; but they are smaller than the uncertainty in the curve fit and therefore can be taken to be zero. Missing data in these Tables are due to premature failure of the specimens from cyclic loading fatigue, as discussed in conjunction with Table 1.

Results in Tables 2 and 3 suggest that at the same frequency and temperature, those materials containing more C-rings exhibit smaller phase lags and lower amplitudes, that is, smaller hole volumes. Unfortunately the paucity of data makes it impossible to conclude definitively that having more mobile moieties, such as C-rings, will suppress the formation of stable nanovoids. Yet the data are consistent with the hypothesis that transient holes arising from external stress relax more rapidly with the insertion of C-rings in the backbone of PET. Chen et al. ${ }^{6}$ found that the activation volume based on the Eyring model increased considerably as the PET based copolymers become more PCT-like, yet the activation energy did not exhibit any significant change. Comparing this observation with the results in Tables 2 and 3, a reasonable explanation is that although the cooperative motion scale becomes larger with the insertion of C-rings in PET's backbone, ${ }^{6}$ the local motion of the backbone also becomes easier and thus faster. Therefore in Chen's case the activation energy did not increase with the activation volume, and in the present case the copolymer containing more Crings in the backbone always exhibits smaller phase lag and amplitude of volume change. Chen et al. hypothesized that the conformational changes of the C-ring reduces the barriers between chain segments to facilitate chain slippage. According to this hypothesis, the formation of stable nanovoids, hence the formation of craze nuclei, is less likely because of relaxation of local dilatative stress. Put another way, at temperatures far below $T_{\mathrm{g}}$, this kind of motion causes the localized dilatative deformation to diffuse as soon as strain is applied, and prevents nascent voids from becoming large stable voids. Consequently these relaxation motions are able to retard the formation of stable craze nuclei, that is, stable nanovoids. The synchronous PALS results in this article are consistent with this hypothesis as well as the findings of $\mathrm{Li}$ and Yee, ${ }^{7}$ and furthermore give some direct information of molecular motion on the microscopic scale.

A note about the surprisingly large effect of temperature is in order. The results presented here, although not entirely conclusive, do suggest that hole relaxation occurs faster at the higher temperature, that is, $25^{\circ} \mathrm{C}$. In the context of the activation energy of the $\gamma$-relaxation in these polyester copolymers, which is between 11 and $15 \mathrm{kcal} / \mathrm{mol}^{6}$ when measured in tension or torsion, the significant difference in the hole amplitude and phase lag

Table 2. Hole Volume Change Under Cyclic Loading At $0{ }^{\circ} \mathrm{C}$

\begin{tabular}{cccc}
\hline C-Ring Content $(\%)$ & PET $(0 \%)$ & EC5 (81\%) & PCT (100\%) \\
\hline Phase lag in degrees & & & \\
10 Hz cyclic loading & $23.5 \pm 3.8$ & - & $15.5 \pm 6.6$ \\
$3 \mathrm{~Hz}$ cyclic loading & $12.0 \pm 4.9$ & - & $3.1 \pm 5.7$ \\
$1 \mathrm{~Hz}$ cyclic loading & $4.1 \pm 5.7$ & - & - \\
Amplitude & & - & $0.053 \pm 0.006$ \\
$10 \mathrm{~Hz}$ cyclic loading & $0.096 \pm 0.006$ & - & $0.039 \pm 0.004$ \\
$3 \mathrm{~Hz}$ cyclic loading & $0.082 \pm 0.007$ & - & - \\
$1 \mathrm{~Hz}$ cyclic loading & $0.04 \pm 0.004$ & & \\
\hline
\end{tabular}


Table 3. Hole Volume Change Under Cyclic Loading at $25 \pm 1.5^{\circ} \mathrm{C}$

\begin{tabular}{lccc}
\hline C-Ring Content $(\%)$ & PET $(0 \%)$ & EC5 $(81 \%)$ & PCT (100\%) \\
\hline Phase lag in degrees & & & \\
10 Hz cyclic loading & $8.6 \pm 3.5$ & $0.7 \pm 4.8$ & $-0.1 \pm 6.0$ \\
3 Hz cyclic loading & $0.2 \pm 4.0$ & $1.8 \pm 5.0$ & $-2 \pm 5.5$ \\
1 Hz cyclic loading & $1.7 \pm 4.9$ & $1.9 \pm 5.9$ & - \\
Amplitude & & & $0.061 \pm 0.006$ \\
10 Hz cyclic loading & $0.081 \pm 0.005$ & $0.056 \pm 0.005$ & $0.047 \pm 0.005$ \\
$3 \mathrm{~Hz}$ cyclic loading & $0.057 \pm 0.004$ & $0.054 \pm 0.005$ & - \\
$1 \mathrm{~Hz}$ cyclic loading & $0.057 \pm 0.005$ & $0.045 \pm 0.005$ & \\
\hline
\end{tabular}

responses of the homopolymers caused by a mere $25{ }^{\circ} \mathrm{C}$ change is surprising. But recall that the change in the ductile-brittle transition temperature upon changing C-ring content is also remarkably large despite the similarity in the activation energies of the $\gamma$-relaxations. ${ }^{6}$ It is quite possible that the dilatative component of the relaxational response of these polymers is different from that of the shear component, a subject that was briefly explored by one of the coauthors. ${ }^{25}$

In closing it must be pointed out that the relatively small amount of results presented here render their interpretation suggestive rather than conclusive. Unfortunately the research cannot be continued because the instruments have been dismantled and the research group dispersed. Nevertheless this article does demonstrate potential for the technique described here to provide fundamental understanding for how molecular motions in the glass are related to the formation of craze nuclei.

This work was supported by a grant from the DMR of NSF, 9971569. The authors thank Eastman Chemical Company for supplying the specimens.

\section{REFERENCES AND NOTES}

1. Boyer, R. F. Polym Eng Sci 1968, 8, 161-185.

2. Heijboer, J. J Polym Sci Part C: Polym Symp 1968, 16, 3755-3763.

3. Sacher, E. J Appl Polym Sci 1975, 19, 1421-1425.

4. Wada, Y.; Kasahara T. J Appl Polym Sci 1967, 11, 1661-1665.

5. Chen, L. P.; Yee, A. F.; Moskala, E. J. Macromolecules 1999, 32, 5944-5955.

6. Chen, L. P.; Yee, A. F.; Goetz, J. M. Schaefer, J. Macromolecules 1998, 31, 5371-5382.

7. Li, L.; Yee, A. F. Macromolecules 2003, 36, 27932801.
8. Liu, J. W.; Yee, A. F. Macromolecules 2000, 33, 13381344.

9. Stevens, J. R. In Methods of Experimental Physics: Polymers; Fava, R. A., Ed.; Academic Press: New York, 1980, Vol. 16, 371ff.

10. Wang, S. J.; Jean, Y. C. Positron and Positronium Chemistry; Shrader, D. M.; Jean, Y. C., Eds.; Elsevier: Amsterdam, 1988; Chapter 8.

11. Shrader, D. M.; Jean, Y. C. Positron and Positronium Chemistry: Shrader, D. M.; Jean, Y. C., Eds.; Elsevier: Amsterdam, 1988; Chapter 3.

12. Deng, Q.; Sundar, C. S.; Jean Y. C. J Phys Chem 1992, 96, 492-495.

13. Wang, Y. Y.; Nakanishi, N.; Jean, Y. C. Sandreczki T. C. J Polym Sci Polym Phys Ed 1990, 28, 1431-1441.

14. Kluin, J.-E.; Yu, Z.; Vleeshouwers, S.; McGervey, J. D.; Jamieson, A. M.; Simha, R.; Sommer, K. Macromolecules 1993, 26, 1853-1861.

15. Hasan, O. A.; Boyce, M. C.; Li, X. S.; Berko, S. J Polym Sci Phys Ed 1993, 31, 185-197.

16. Xie, L.; Gidley D. W.; Hristov, H. A.; Yee, A. F. J Polym Sci Phys Ed 1995, 33, 77-84.

17. Ruan, M. Y.; Moaddel, H.; Jamieson, A. M.; Simha, R.; McGervey, J. D. Macromolecules 1992, 25, 2407-2411.

18. Eldrup, M.; Lightbody, D.; Sherwood, J. N. Chem Phys 1981, 63, 51-58.

19. Nakanishi, H.; Ujihira, Y. J Phys Chem 1982, 86, 4446-4450.

20. Nakanishi, H. In Proceedings of the Conference on Positron Annihilation in Liquids, Arlingto, TX; Sharma, S. C., Ed.; World Scientific: Singapore, 1987; p 292.

21. Opp, D. A.; Skinner, D. W.; Wiktorek, R. J. Polym Eng Sci 1969, 9, 121-130.

22. Schrager, M. J Polym Sci A-2: Polym Phys 1970, 8, 1999-2014.

23. Koo, G. P.; Riddell, M. N.; O’Toole, J. L. Polym Eng Sci 1967, 7, 182-188.

24. Zilvar, V. Plast Polym 1971, 39, 328-332.

25. Yee, A. F.; Takemori, M. T. J Polym Sci Polym Phys Ed 1982, 20, 205-224. 